\title{
Una visión de la Delegación del CSIC en Cataluña
}

Joan Albaigés *

Arbor CLXXVI, 695-696 (Noviembre-Diciembre 2003), 179-190 pp.

Las Delegaciones territoriales del CSIC pueden ser, no sólo unidades de coordinación y representación institucional, sino también instrumentos idóneos para la búsqueda de oportunidades de implantación del Organismo en todo el Estado. En el presente artículo se describe la evolución del papel que la Delegación de Cataluña ha tenido a lo largo de sus 60 años de existencia y se hace especial hincapié en la necesidad de superar las dinámicas de concurrencia de iniciativas con el establecimiento de fórmulas de concertación, reconociendo la existencia de especificidades territoriales. Éstas pueden implicar estructuras organizativas diversas, como las que se ilustran en el artículo, para el caso de Cataluña, que pueden enriquecer y fortalecer el panorama investigador.

A lo largo de sus más de seis décadas de existencia, la Delegación del CSIC en Cataluña ha sido reflejo y testimonio de la evolución, no sólo de nuestra ciencia, sino también de nuestro entorno social y político, y se encuentra, hoy día, ante nuevos retos y posibilidades, sobre los cuales convendría reflexionar. El presente artículo pretende aproximarse a algunas cuestiones que se perciben de forma específica en Cataluña y que ilustran las distintas vertientes de la política científica en nuestro país. 


\section{Antecedentes históricos}

La creación de una Delegación del CSIC en Barcelona, a principios de los años 40, representa el primer intento de extender fuera de Madrid las actividades del Organismo, creado con el objetivo de «fomentar, orientar y coordinar las dispersas muestras de la investigación científica en España» y, de esta forma, contribuir a «la restauración de la clásica y cristiana unidad de las ciencias, destruida en el siglo XVIII». Tan loable propósito de descentralización requirió, sin embargo, la modificación de la Ley Fundacional de 1939, que formalmente se aprobó el 21 de noviembre de 1942.

Desde un principio la Delegación albergó, además de los órganos administrativos (presidencia, secretaria, etc.), las Secciones de Humanidades, dejando las actividades relacionadas con las ciencias experimentales en el entorno de las cátedras universitarias. Hay que recordar que, en aquella época, el CSIC ofrecía solamente cobijo científico y un mínimo soporte económico a las actividades de sus miembros, generalmente profesores universitarios, por lo que la organización institucional tenía un marcado componente individual.

En este sentido, es difícil encontrar estudiosos, que en los años 50 y 60 dejaron su huella tanto en el ámbito de las ciencias experimentales como en el de las humanidades, que no tuvieran alguna relación con el CSIC. De hecho, acogió una parte importante de la élite intelectual catalana que había sobrevivido a la dramática depuración del régimen franquista, con figuras de tanto prestigio como Jordi Rubió i Balaguer, Jaume Vicens Vives, Higini Anglés, Tomás Carreras i Artau, Pius Font i Quer, Josep Pascual Vila, Ramón Trias Fargas, etc... En realidad, como han señalado varios autores ${ }^{1}$, la dinámica interna de la Institución no se correspondía con su propia Norma, pero ello no impidió que la imagen de una Institución integrante del aparato ideológico del franquismo lastrara considerablemente su proyección pública, hasta bien entrada la democracia.

No deja de ser indicativo, a este respecto, que para ubicar su sede se escogiera el corazón de uno de los entornos urbanos más representativos de la cultura catalana de la época republicana, donde se encontraban la Biblioteca Nacional de Cataluña y el Institut d'Estudis Catalans, la primera convertida en Biblioteca Central y el segundo declarado fuera de la ley.

El edificio de la Delegación, inaugurado en diciembre de 1954 en la calle Egipcíacas, se construyó de estilo similar a las históricas construcciones vecinas, uniéndolo mediante un controvertido puente con 


\section{Una visión de la Delegación del CSIC en Cataluña}

la antigua sede del IEC, en un simbólico intento de ocupación de las Instituciones históricas de Cataluña, abolidas por la dictadura.

En 1963, la Delegación del CSIC en Barcelona tenía inscritas 76 Secciones, correspondientes a 47 Institutos, que dependían de los siete Patronatos que entonces configuraban el Consejo. Una estructura impresionante sobre el papel, pero sin base real, ya que muchos de estos Institutos o Secciones eran puramente nominales, con una muy baja producción y casi nula actividad institucional. Los pocos referentes que quedan en mi memoria de la Delegación de finales de los 60 son las visitas a final de mes para cobrar la nómina y las solemnes celebraciones de la festividad de San Isidoro. Realmente, aparte de ciertas labores administrativas y de representación, sus funciones eran muy limitadas.

\section{La Delegación, a partir de los 80}

A pesar de los profundos cambios habidos en nuestro país a mediados de los 70, éstos no alcanzan a la política científica hasta casi diez años más tarde. Es en la década de los 80 cuando se ponen unas nuevas bases, que tienen su principal expresión en la Ley de la Ciencia de 1986. La década de los ochenta se caracterizó por un crecimiento considerable, tanto cuantitativo como cualitativo, del Sistema Español de Ciencia y Tecnología, impulsado inicialmente por la puesta en marcha del Plan Nacional y, posteriormente, por la incidencia de los programas europeos.

Coincide este período con la llegada de Enrique Trillas a la Presidencia del CSIC que, con un potente e imaginativo equipo de gobierno, inicia una profunda reconversión del Organismo. «El CSIC, que nació como un producto de la guerra civil, enfrentado a todo lo que tuviera algo de liberal, quiere ahora realizar una labor de reparación histórica, para impulsar un futuro inteligente», afirmaba Trillas en la presentación del primer congreso sobre la Junta de Ampliación de Estudios ${ }^{2}$. Pero no es a esta tarea, cuyo análisis y valoración ya figura en otra parte de este volumen, a la que nos vamos a referir sino a su proyección en la nueva concepción de la Delegación.

La primera decisión fue transformar la Delegación de Barcelona en Delegación de Cataluña, en sintonía con la nueva estructura político-administrativa del Estado, al frente de la cual situó a Josefina Castellví. La Delegación empezó a ser punto de encuentro de los Directores de los distintos Institutos de Cataluña, a jugar un creciente 
papel en las relaciones con las instituciones locales, y a percibirse una cierta recuperación de su imagen corporativa. La existencia de amplios espacios infrautilizados en el propio edificio de la Delegación, permitió abrir sus puertas a instituciones tan diversas como la Secretaría de la UIMP en Cataluña, la Gerencia del Museo de Arte Contemporáneo de Barcelona (MACBA), el Centro de Información y Documentación Internacional de Barcelona (CIDOB), la Sociedad Catalana de Documentación Informatizada (SOCADI) o el Comité de los Voluntarios de los JJOO del 92.

Lo cierto es que el CSIC en Cataluña logró rentabilizar de forma especialmente positiva los nuevos aires que soplaban en la ciencia española. La creación de nuevos Institutos, principalmente en el campus de la UAB, también contribuyó, no sólo a normalizar su situación, sino a hacerse un lugar en el panorama investigador y universitario.

La construcción, en 1993, de una nueva Residencia de Investigadores en el edificio de Egipcíacas, gracias a un acuerdo con la Generalitat de Cataluña, venía a completar de forma satisfactoria el marco de cooperación institucional con el Gobierno Autónomo, ofreciendo una plataforma conjunta de acogida para científicos, así como de numerosas actividades científico-culturales en colaboración con otras Instituciones. La Residencia, además, se constituyó bajo la figura de un Consorcio, una figura particularmente adecuada para la gestión compartida de iniciativas, que, desgraciadamente, no ha sido utilizada con mayor frecuencia y a la que nos referiremos más adelante.

Sin embargo, a mediados de los años 90, la favorable evolución en la financiación y en la reordenación del sistema científico español sufre una evidente inflexión. A partir de entonces, el crecimiento de los presupuestos del CSIC se estanca y el proceso de reformas se desacelera, a pesar de la positiva experiencia acumulada. Un ejemplo de esta paralización fue la redacción del reglamento de funcionamiento interno, acorde con la Ley de la Ciencia, que requirió diez años y decenas de borradores antes de su aprobación. En dicho reglamento se perdió una gran oportunidad para abordar diversos aspectos organizativos, entre ellos la organización territorial del Organismo, para buscar y potenciar aquellas sinergias que le permitieran mejorar su capacidad competitiva. Resulta significativo que los Delegados territoriales pasaran a denominarse Coordinadores institucionales, con funciones meramente representativas ante los diferentes organismos e instituciones públicas y privadas del ámbito territorial.

En Cataluña, además, la positiva tendencia del CSIC se vio afectada por dos circunstancias coyunturales. En estos años tuvo lugar la trans- 


\section{Una visión de la Delegación del CSIC en Cataluña}

ferencia de competencias en materia de enseñanza universitaria a la Generalitat de Cataluña, con lo que las inversiones en instalaciones y dotaciones universitarias pasaron a ser la principal prioridad política. Por otra parte, el gobierno catalán planteó un conflicto de competencias ante el Tribunal Constitucional, al entender que la Ley de la Ciencia vulneraba, en materia de investigación, el Estatuto de Cataluña.

Como es sabido, el Tribunal desestimó el recurso con el argumento de que se trataba de competencias «concurrentes» entre el Estado y las CCAA, frente a lo que hubiera podido ser una interpretación más compartida de responsabilidades, según el criterio de conocidos expertos constitucionalistas ${ }^{3}$. En el fallo del alto tribunal se llegaba a decir que «aunque sería deseable que el ejercicio de las competencias estatales y autonómicas en materia de investigación no tuviera por efecto la duplicación, la descoordinación o el despilfarro, no por ello tendría que traducirse en un tipo de articulación distinta a la propuesta (en la Ley)».

En todo caso, la corrección jurídica de la Ley, apreciada por el T.C., no eximía de la responsabilidad política de buscar planteamientos de mayor racionalidad y eficiencia. En este sentido, una interpretación más abierta de los ámbitos competenciales hubiera podido permitir el establecimiento de una combinación concertada de agentes e instrumentos a los niveles apropiados, sobre la base de una corresponsabilidad en la toma de decisiones, gestión y financiación de la $\mathrm{I}+\mathrm{D}$, que hubiera rendido indudables beneficios al sistema. Sin embargo, el resultado fue la existencia de dos políticas diferenciadas y descoordinadas de investigación, con incidencia en el mismo territorio, en ejercicio de dos competencias que, bajo el concepto de concurrentes, parecían obviar los criterios de eficiencia en la gestión de los recursos públicos.

Este problema fue objeto de un amplio debate en distintos medios y condicionó, sin duda, algunas decisiones y actuaciones de los distintos actores. Por aquel entonces escribía: «Entendemos que la política científica debería comprender una distribución de competencias entre las distintas administraciones, ni excluyentes ni idénticas, sino complementarias o compartidas, desde los primeros niveles de planificación, organización y fomento de la investigación hasta los de gestión y ejecución. La competencia de propuestas, tan importante para mejorar la calidad de la actividad científica de los equipos de investigación, puede resultar negativa llevada a los primeros niveles de la planificación y organización de la misma» ${ }^{4}$.

En este contexto, resultaba fundamental el diálogo y la voluntad política de cooperación institucional, especialmente en un período de 
escasez de recursos. Por su parte, la Delegación, con sus limitaciones, era un observatorio privilegiado de las oportunidades de crecimiento y consolidación que podía tener el CSIC en Cataluña y podía constituir un instrumento de concertación idóneo. Pero la escasa voluntad de salirse de los parámetros organizativos convencionales, homogéneos para todo el Organismo, y el temor del MEC a «perder la propiedad» del único organismo de ámbito estatal que le quedaba, una vez completadas las transferencias universitarias a las CCAA, fueron un freno para asociarse a algunas iniciativas que se estaban poniendo en marcha. Por la importancia que éstas pueden tener en el futuro, merece la pena extendernos en algunas consideraciones.

\section{Algunas consideraciones sobre la coordinación institucional}

$\mathrm{Al}$ analizar las expectativas de crecimiento del Organismo, se corre el riesgo de pensar que todo se reduce a un problema de financiación, o de necesidad de mayores recursos para el sistema, cuando en realidad un aumento de los mismos será ineficaz si no encuentra un entramado organizativo, suficientemente consistente a todos los niveles, desde la planificación a la gestión, como para ser capaz de generar los retornos que la sociedad legítimamente espera obtener de su esfuerzo.

De la misma forma que el incremento substancial de recursos públicos destinados a la investigación en España en la década de los 80, fue acompañado de la creación de un sistema competitivo para la obtención de los mismos, que logró un importante incremento no sólo cuantitativo sino también cualitativo de la actividad investigadora, el nuevo salto que ahora se requeriría obliga a implementar nuevos instrumentos o a adoptar nuevas estrategias para corregir los desajustes existentes.

Ciertamente, se podrá esgrimir que la propia dinámica de un sistema competitivo como el actual, simplemente con mayores recursos en juego, sería suficiente para situarnos donde pretendemos estar. Pero conviene recordar que el conocimiento o progreso científico, que se concreta en innovación y ésta en progreso social y crecimiento económico, no es una secuencia de desarrollo lineal, sino que diversos condicionantes de tipo estructural o estratégico, conducen a resultados distintos, a igualdad de esfuerzo financiero.

En el análisis de la situación actual podríamos hablar de la excesiva fragmentación de los grupos de investigación, de su reducido tamaño, de la escasa incidencia que la demanda ha tenido en su creación y, 


\section{Una visión de la Delegación del CSIC en Cataluña}

en consecuencia, de su escasa capacidad innovadora. Podríamos hablar también de una excesiva concentración territorial del esfuerzo público y de una escasa eficiencia del sistema de financiación en términos de innovación productiva. Evidentemente, la mayoría de estas deficiencias, que definen la debilidad de nuestro sistema de I+D, tienen su origen en el bajo nivel de inversión pública, pero no sólo en él. Hay problemas de tipo financiero, pero también los hay de tipo estructural y organizativo, que convendría abordar desde planteamientos políticos renovados.

Conviene recordar, también, que el establecimiento de entornos competitivos tiene un fuerte componente territorial. La capacidad innovadora de un territorio y las diferencias en su desarrollo, atribuibles a la variable $\mathrm{I}+\mathrm{D}$, están hoy perfectamente identificadas y es por ello que en los estudios de economía industrial se habla ya, con pleno fundamento, de Sistemas territoriales de Ciencia y Tecnología. Sistemas que pueden proporcionar determinados elementos de soporte y ventajas competitivas para los sectores productivos, y especialmente para los innovadores, que son los capaces de generar nuevos sectores y contribuir en mayor medida al crecịmiento económico.

No es necesario acudir a ejemplos foráneos, basta con analizar las ratios de producción científica y de captación de recursos competitivos en el sector público, o de registros de patentes europeas por recursos invertidos en el sector privado, en las distintas CCAA, para darnos cuenta de que no existe una relación directa entre el esfuerzo inversor en $\mathrm{I}+\mathrm{D}$ y su rendimiento. El entorno es un factor determinante $\mathrm{y}$ éste puede organizarse y gestionarse mucho mejor desde una mayor proximidad.

El análisis de las deficiencias generales del sistema y de las especificidades territoriales son hoy elementos fundamentales para la definición de los planes futuros de actuación, que pueden recibir un mejor impulso en un marco de coordinación institucional. La búsqueda de fórmulas imaginativas y eficaces para una mejor organización y gestión del sistema de $\mathrm{I}+\mathrm{D}$ han sido y son una constante en Cataluña y sería bueno, desde los distintos ámbitos de responsabilidad, promover puntos de encuentro. No se trata de inventar nada nuevo. Modelos similares de corresponsabilidad en los distintos niveles de la política científica existen en el mundo desarrollado. No deja de ser significativo que, en Europa, sean precisamente los países con un modelo más descentralizado y concertado los que han alcanzado un mayor nivel de eficiencia investigadora y capacidad innovadora. Es el caso de Alemania, Bélgica y Suiza, todos ellos con contribuciones superiores al 1,5\% del 


\section{Joan Albaigés}

PIB, y con contribuciones de los sectores industriales superiores al 60-70\%.

El propio reglamento de organización y funcionamiento del CSIC reconoce un amplio campo de posibilidades de cooperación institucional. En concreto, en el artículo 41 indica que:

«El CSIC podrá establecer otras modalidades de colaboración institucional, para atender nuevas necesidades o demandas que puedan producirse en la evolución del contexto de sus relaciones institucionales».

Con el fin de profundizar en ellas, se examinan a continuación algunas figuras especialmente idóneas para mejorar la eficiencia del propio sistema.

\section{La figura consorcial}

El consorcio es un instrumento idóneo para la cooperación interinstitucional. En los últimos años, esta figura ha sido muy utilizada con tal objetivo, ya sea para atender finalidades de cooperación en el ámbito de las obras y servicios o de cooperación entre diversas administraciones públicas en materia de competencias compartidas o concurrentes.

Por su gran flexibilidad y por su facilidad para incorporar representantes de instituciones diversas en finalidades de interés común, el consorcio es una técnica de cooperación de utilidad creciente en nuestro marco institucional. Expertos administrativistas han destacado su rigurosa modernidad, operatividad y eficacia ${ }^{5}$ y, en Cataluña, tiene una gran tradición en diversos sectores, incluidos la investigación y el desarrollo tecnológico. Algunos Centros de reconocido prestigio, como el Centro de Estudios Demográficos, el Instituto de Física de Altas Energías, el Centro Internacional de Métodos Numéricos (CIMNE), el Centro de Investigación Ecológica y Recursos Forestales (CREAF), etc., se crearon adoptando este modelo organizativo.

La actual distribución de competencias en $\mathrm{I}+\mathrm{D}$, con la superposición de estructuras y actuaciones de las Administraciones central y autonómica, sin un marco adecuado de concertación, conduce, a menudo, al solapamiento de esfuerzos, a la duplicación de servicios y al cansancio del investigador, que debe redoblar sus esfuerzos en busca de la solución de sus problemas. 
La figura del Consorcio permite orientar este esfuerzo en una única dirección bien planificada y, al mismo tiempo, teniendo en cuenta que en él están representadas las diferentes Administraciones competentes, permite asegurar la coherencia de todo el sistema, desde la planificación o definición de objetivos hasta la ejecución y seguimiento de las actuaciones. Un aspecto no menos importante que conviene destacar de la fórmula consorciada es el de facilitar el acercamiento entre la oferta pública de investigación y la demanda de sectores productivos, o incluso participar en iniciativas empresariales.

Asimismo, el Consorcio permite potenciar la actividad investigadora de los entes consorciados, ya que la investigación que realiza puede considerarse parte integrante de sus respectivas programaciones o planes estratégicos.

Finalmente, la posibilidad de adoptar formas ágiles de gestión redunda en beneficio de las prestaciones y eficacia de la labor de los centros, sin detrimento de que, a través de sus estatutos, se establezcan los necesarios mecanismos de control.

La norma constitutiva y carta de naturaleza de los consorcios son sus Estatutos. La aprobación de los Estatutos señala el momento constitutivo del consorcio y su contenido determina, entre otros: su denominación, composición, objetivos, régimen aplicable, organización, financiación, etc.

Los Estatutos del consorcio deben prever su estructura organizativa y la correspondiente participación de los representantes de las entidades consorciadas en los órganos de gobierno. Los estatutos tienen plena libertad configuradora de la estructura organizativa. Lo normal es que exista, como mínimo, un órgano colegiado representativo de todas las entidades consorciadas (plenario, comisión gestora) y los correspondientes órganos de carácter unipersonal con responsabilidades de representación y gestión del consorcio (presidente, gerente).

Los Estatutos también deben prever de que forma han de ser financiadas las actividades a desarrollar por el consorcio. Es normal que, al menos en parte, la financiación dependa de aportaciones de las entidades consorciadas, cuyos criterios de participación pueden especificarse en los estatutos. Asimismo, es necesario señalar que el consorcio tiene capacidad para gestionar la recaudación de los ingresos generados por sus actividades, pudiendo incluso utilizar el procedimiento de apremio.

No existe ninguna regulación general sobre el procedimiento a seguir para decidir la constitución de un consorcio ni para la elaboración y aprobación de sus estatutos, sino que dicho procedimiento estará en función de cada tipo de administración a consorciar. 
La Ley 4/1999 contiene algunas referencias generales a los Consorcios, $y$ hace referencia directamente a los consorcios que se constituyen para gestionar convenios de colaboración entre el Estado y las Comunidades Autónomas. El artículo 6 de esta Ley, en su apartado 5, especifica:

"Cuando la gestión del convenio haga necesario crear una organización común, ésta podrá adoptar la forma de consorcio dotado de personalidad jurídica o sociedad mercantil.

Los estatutos del consorcio determinarán los fines del mismo, así como las particularidades del régimen orgánico, funcional y financiero.

Los órganos de decisión estarán integrados por representantes de todas las entidades consorciadas, en la proporción que fije en los Estatutos respectivos.

Para la gestión de los servicios que se le encomienden podrán utilizarse cualquiera de las formas previstas en la legislación aplicable a las Administraciones consorciadas."

Por su parte, la legislación administrativa catalana también proporciona cobertura legal a los consorcios en el artículo 55 de la Ley 13/1989, que dice:

«El Gobierno puede acordar la constitución de consorcios entre la Generalitat $y$ otras administraciones públicas para finalidades de interés común o con entidades privadas sin ánimo de lucro que tengan finalidades públicas concurrentes con las de la Administración.

Los consorcios tienen personalidad jurídica propia.

Los estatutos de los consorcios han de determinar las finalidades y las particularidades de los regímenes orgánico, funcional y financiero.

Los órganos de decisión de los consorcios han de estar integrados por representantes de todas las entidades consorciadas, en la proporción que se fije en los estatutos respectivos.»

Aunque hasta el presente las iniciativas hayan sido escasas, no por ello deben dejarse de contemplar en el futuro. Tras la primera experiencia con la Residencia de Investigadores, recientemente se aprobó el Instituto de Biotecnología Vegetal, como Consorcio entre el CSIC y el IRTA. Significativa ha sido también la puesta en marcha del Consorcio para la construcción y explotación del Laboratorio de Luz de Sincrotrón, como un ente de derecho público integrado por la Administración General del Estado y la Generalitat de Cataluña. 


\section{La figura fundacional}

Las fundaciones se conocen como entidades sin ánimo de lucro, constituidas por personas físicas o jurídicas, mediante la afección de unos bienes o derechos con vistas a unas finalidades de interés general. Para constituir una Fundación hay que manifestar la voluntad de los fundadores en escritura pública y la personalidad jurídica se adquiere con su inscripción en el registro de Fundaciones.

La Ley catalana de fundaciones, de 2 de Mayo de 2001, recoge la posibilidad de que personas jurídicas públicas puedan también constituir fundaciones. De esta forma, desde la Generalitat se han impulsado, recientemente, diversas fundaciones de promoción y control público que tienen como objetivo principal actividades de I+D.

La decisión de potenciar esta figura se basa en el hecho de que se trata de entes especialmente pensados para implicar a sectores económicos y productivos en sus objetivos, teniendo en cuenta que estos sectores se benefician de importantes ventajas de tipo fiscal y de un especial tratamiento jurídico. Estas ventajas, junto con otras de orden menor pero también importantes, como puede ser la facilidad para su constitución y gestión, las convierten en un instrumento especialmente adecuado a las finalidades propias de la I+D y de la captación de intereses de todo tipo relacionados con la innovación y la transferencia de tecnología.

Algunos Centros de investigación creados recientemente bajo la figura de una fundación son el Centro de Regulación Genómica, el Parque de Investigación Biomédica de Barcelona, el Instituto Catalán de Nanotecnologías, el Instituto Catalán de Investigación Química, en Tarragona, etc.

Merece la pena destacar que, tanto en el caso de los consorcios como de las fundaciones, la actuación de la Administración se sitúa en el nivel de la planificación, fomento y control a posteriori, dejando la ejecución y gestión diaria en manos de los principales actores, para lo cual se les dota de personalidad jurídica propia.

En base a esta jurisprudencia podrían considerarse diversos escenarios para el desarrollo de una actuación concertada del CSIC en Cataluña, desde un planteamiento global, que podría abarcar todos los centros, hasta actuaciones puntuales, en función de la naturaleza de sus actividades. Fórmulas existen muchas e impedimentos jurídicos-administrativos muy pocos.

\section{Conclusión}

Más que «una visión de la Delegación del CSIC en Cataluña» este artículo bien podía haberse titulado «reflexiones sobre el CSIC desde 
la periferia"». En cualquier caso, desde la Delegación se pueden percibir caras distintas de una misma realidad que conviene analizar con rigor. En este sentido, una política científica coherente y con voluntad de contribuir a resolver los grandes retos que nuestra sociedad y nuestra economía tienen planteados en estos inicios de siglo, debería prestar atención, entre otros, a los problemas que acabamos de describir.

La introducción en el Sistema de fórmulas de concertación, podría contribuir, sin duda, a corregir los desajustes existentes y a incorporar una pluralidad de planteamientos que enriquecerían y fortalecerían el panorama investigador. En un modelo de este tipo, la capacidad directiva o de liderazgo del Organismo tendría precisamente un papel principal, pudiendo ejercer, además, funciones de validación o reconocimiento de la excelencia investigadora de las iniciativas concertadas.

Que la Ciencia española necesita un período de reflexión y nuevas ideas es algo reconocido por todos. Esperemos que, por su bien, se concreten en un futuro próximo.

\section{Notas}

* Ex-Delegado del CSIC en Cataluña. Ex-Comisionado de Universidades e Investigación de la Generalitat de Cataluña.

1 L. Calvo y J. Josa, "La Ciència a Catalunya: avatars en el món científic», en Història de la cultura catalana, Ed. 62 (Barcelona), 1998.

L. Calvo, La Delegación y los Centros del CSIC en Cataluña: sesenta años al servicio de la Ciencia. CSIC (Barcelona), 2002.

2 La Junta de Ampliación de Estudios e Investigaciones Científicas. J.M. Sanchez Ron (ed.). CSIC, Madrid, p. VIII (1988).

3 A. Milian. "Las competencias del Estado y de las CCAA, con especial referencia a Cataluña, en materia de investigación científica y técnica: ¿Concurrencia o compartición?. En «El paper de les CCAA en l'ordenació i gestió del sistema universitari i la política de recerca». Col. Institut d'Estudis Atonòmics, 37 (2002).

4 J. Albaigés, Política científica y cooperación institucional. El País, 23 Febrero 1994.

5 R. Martín Mateo, “Los consorcios locales. Una institución en auge». Revista de Administración Pública, 129, 1992. 